Article

\title{
An Effective Delivery System of Sitagliptin Using Optimized Mucoadhesive Nanoparticles
}

\author{
Afzal Haq Asif ${ }^{1, *}$, Sree Harsha ${ }^{2, *(D)}$, Niranjan Hodalur Puttaswamy ${ }^{3}$ and \\ Bandar E. Al-Dhubiab ${ }^{2}$ \\ 1 Department of Pharmacy Practice, College of Clinical Pharmacy, King Faisal University, \\ Al-Ahsa 31982, Saudi Arabia \\ 2 Department of Pharmaceutical Sciences, College of Clinical Pharmacy, King Faisal University, \\ Al-Ahsa 31982, Saudi Arabia; baldhubiab@kfu.edu.sa \\ 3 Department of Biomedical Sciences, College of Medicine, King Faisal University, \\ Al-Ahsa 31982, Saudi Arabia; niranjanhp@gmail.com \\ * Correspondence: ahasif@kfu.edu.sa (A.H.A.); sharsha@kfu.edu.sa (S.H.); Tel.: +96-653-548-5322 (S.H.)
}

Received: 12 May 2018; Accepted: 21 May 2018; Published: 24 May 2018

Abstract: Sitagliptin (MK-0431), is a potent oral hypoglycemic drug that is used for treating type 2 diabetes mellitus. However, the short half-life of sitagliptin requires patients to take a high dose of $50 \mathrm{mg}$ twice per day, and the fraction of sitagliptin reversibly bound to plasma proteins is as low as $38 \%$. In addition, it was reported that approximately $79 \%$ of sitagliptin is excreted unchanged in the urine for elimination without metabolism. Thus, a better delivery system is needed to improve the benefits of sitagliptin in patients. The drug content and percentage yield were found to be $73 \pm 2 \%$ and $92 \pm 2 \%$, respectively. The optimized sitagliptin nanoparticle sizes were between 350-950 nm, and the surfaces were smooth and nearly spherical in shape. In addition, the optimized sitagliptin nanoparticles have an indicated excellent bioadhesion property of (6.1 $\pm 0.5 \mathrm{~h})$. The swelling of the nanoparticles is $168 \pm 15 \%$. The pattern of sitagliptin release from the mucoadhesive nanoparticles follows the Korsmeyer-Peppas model. More importantly, the extended sitagliptin retention time, of up to $12 \mathrm{~h}$ in the gastrointestinal tract, suggests that the optimized mucoadhesive nanoparticle formulation is more efficient, and has a greater potential to be used for oral delivery compared to the conventional sitagliptin administration in the drug solution. This is the first developed delivery system using the optimized mucoadhesive nanoparticles to enhance the effectiveness of sitagliptin.

Keywords: sitagliptin; diabetes mellitus; nanoparticle; mucoadhesive

\section{Introduction}

Diabetes mellitus (DM) is a type of metabolic disorder with a high level of blood glucose over a prolonged period of time. Diabetes is rapidly increasing in every single part of the world and is considered as one of the major risks to public health in the 21st century. It is expected that the prevalence of the DM will affect 366 million people by 2030 globally [1-3], with $90 \%$ of cases being the type 2 form of the disease [4].

International Diabetes Federation data indicate that nine countries across the Gulf Region have the highest adult diabetes population, including Oman (13.4\%); United Arab Emirates (18.7\%); Saudi Arabia (16.8\%); Kuwait (14.6\%); Mauritius (16.2\%); Bahrain (15.4\%); Tonga (13.4\%); Malaysia (11.6\%); and Reunion (15.3\%) [5]. Saudi Arabia is a developing country that has a population of over 28 million [6], of which 25\% has been diagnosed with DM [7]. In addition, an increase of $40-50 \%$ by 2020 is also predicted [8]. 
The current oral antidiabetic agents have no significant effect on repairing the actual secretory function of the pancreatic islet cells, and are often limited by many side effects, including hypoglycemia, dizziness, and nausea during clinical treatment. As a result, symptoms of Type 2 diabetes mellitus (T2DM) patients remain incompetently managed. Thus, there has been an extensive interest in recognizing or identifying newer therapeutics for treating T2DM [9].

The dipeptidyl peptidase-4 (DPP-4) inhibitors are a relatively new class of oral diabetes drugs available for the treatment of type 2 diabetes mellitus (T2DM). Strategies for treatment include DPP-4 inhibition, as well as to raise circulating levels of the incretin hormones that are involved in decreasing blood glucose levels in the blood, glucagon-like peptide-1 (GLP-1) and glucose-dependent insulinotropic polypeptide (GIP). Sitagliptin (LC15-0444), a newly developed potent oral anti-hyperglycemia agent (anti-diabetic drug) DPP-4 inhibitor, was extensively studied in clinical trials to investigate the molecular interaction with sitagliptin and type 2 diabetes enzyme (DPP-4) [10]. It was approved by the Korea Food \& Drug Administration (KFDA) in the year 2012. It is well known that the blood glucose-lowering effects are mediated by glucagon-like peptide 1GLP-1 and the gastric inhibitory polypeptide (GIP) incretin hormones that are inactivated by DPP-4. Unfortunately, one of the primary weaknesses of using sitagliptin is its biological half-life of $3.6 \mathrm{~h}$ in rats, and it is eliminated rapidly. Due to the shorter half-life of sitagliptin, it is suggested that patients need to follow, strictly, the dosing interval, which requires sitagliptin to be administered in two doses of $50 \mathrm{mg}$ per day [11-13].

Albumin has excellent mucoadhesive and $\mathrm{pH}$-sensitive properties with controlled drug release characteristics, and thus, has been used widely to increase the delivery of active drugs into multiple mucosal sites [14]. Mucoadhesive drug delivery system offers several benefits by targeting and localization of the dosage form at a specific site, including the minimization of fluctuations in drug concentrations in the plasma, and prolonged retention time at the site of drug absorption, and a strengthened contact of therapeutic agent with the mucosa to increase the drug concentration gradient and also prevent adverse effects. Therefore, absorption and subsequent bioavailability of drugs may be improved, and reducing dosage intervals results in better patient compliance $[15,16]$.

The aim of this research is to optimize a mucoadhesive nanoparticle formulation for the delivery of sitagliptin nanoparticles, and evaluate the in vivo drug distribution and retention in rat gastrointestinal tract (GIT). This is the first mucoadhesive nanoparticle drug delivery system that was ever designed to increase the efficacy of the antidiabetic drug sitagliptin.

\section{Material and Methods}

Sitagliptin, sodium deoxycholate $\geq 97 \%$, and membrane filter $(0.22 \mu \mathrm{m})$ were purchased from Sigma Aldrich Co. (St Louis, MO, USA). All other chemicals and reagent used were of HPLC analytical grade. Water was double distilled by a Milli-Q Reagent Grade (Millipore-Corporation, Bedford, MA, USA).

\subsection{Formulation of Sitagliptin Nanoparticles}

Polymer-loaded sitagliptin nanoparticles were prepared by nano spray dryer method (BUCHI Labortechnik AG). The spray dryer contains a pulsating casing in the spray nozzle to atomize the feed, and an electrostatic particle accumulator gathered the particles. The spray mesh size of $4 \mu \mathrm{m}$ was used, the feed flow rate was set to about $100-120 \mathrm{~L} / \mathrm{min}$, the relative spray rate was fixed to $50 \%$, and inlet temperature $\left(120^{\circ} \mathrm{C}\right)$ and outlet temperature $\left(30^{\circ} \mathrm{C}\right)$ were established. A solution of albumin (500 mg albumin A soaked for $60 \mathrm{~min}$ in water and warmed before spraying) containing the same amount of sitagliptin was also spray dried. The solid particles collected from drying chamber using a spatula were stored at room temperature. 


\subsection{Characterization of Nanoparticles}

\subsubsection{Scanning Electron Microscopy (SEM) of Nanoparticles}

The uncoated sitagliptin nanoparticles were examined using different magnifications $(1000 \times-95,000 \times)$ by scanning electron microscopy (Jeol Analytical Scanning Microscope, JSM-6390LA, Tokyo, Japan). Different batches of nanoparticles were assessed after platinum sputtering (Jeol JFC-1600, Tokyo, Japan).

\subsubsection{Analysis of Particle Size of Nanoparticles}

The particle size were measured by Malvern Zetasizer Nano ZS, Malvern Instruments Ltd. Worcestershire WR14 1XZ, United Kingdom. The sample were dispersed in a liquid medium, i.e., in absolute ethanol with refractive index of 1.33 and the suspension were measured at $25^{\circ} \mathrm{C}$.

\subsubsection{Swelling Measurement}

The swelling of nanoparticles was assessed in ethanol. The sizes of dried nanoparticles were measured using microscopy after incubation in ethanol for $0.15,0.25,0.5,1.0,3.0,5.0,8.0$, and $12.0 \mathrm{~h}$. The swelling percentage were determined from the below formula, time " $t$ " (Gt) and the initial time $(t=0[\mathrm{G} 0])$ as calculated as below.

$$
\text { Swelling } \%=\text { Gt }- \text { G0/G0 × } 100
$$

\subsubsection{Production Yield}

Nanoparticle yield was determined after collecting the particles from the spray drying chamber and calculated by the below formula:

Percentage Yield $=($ Practical yield $) /($ Total weight of the polymer plus drug $) \times 100$

\subsubsection{In Vitro Wash-off Test for Nanoparticles}

The mucoadhesive properties of nanoparticles were determined using a reported technique as previously described (Rao and Buri, 1989). Briefly, rats were anesthetized with isoflurane $/ \mathrm{O}_{2}$, and stomach mucosa (about $5 \mathrm{~cm}$ and $1 \mathrm{~cm}$ wide) were excised and rinsed with normal saline. A $100 \mathrm{mg}$ amount of the formulation (equivalent to drug) was placed on the stomach mucosal surface and allowed to react for $30 \mathrm{~min}$. The mucosal piece was then kept in a desiccator for $15 \mathrm{~min}$ with $90 \%$ relative humidity. The mucosa was then thoroughly washed using a peristaltic pump using saline (flow rate of $5 \mathrm{~mL} / \mathrm{min}$ ). The concentration of the drug was determined spectroscopically.

\subsubsection{Nanoparticle Sitagliptin Drug Release}

Drug release pattern was assessed using a dialysis method. Sitagliptin nanoparticles were packed in a dialysis tubing membrane with high retention cellulose tubing of 12,400 membrane molecular weight cutoff (MWCO) in a mixture of $200 \mathrm{~mL}$ of phosphate buffered saline (PBS), and sodium deoxycholate $-2 \%$ ( $\mathrm{pH} 6.8 \pm 0.2$ ) sink condition was maintained. The PBS was then topped up to $1 \mathrm{~L}$ with $800 \mathrm{~mL}$ water and the $\mathrm{pH}$ was adjusted to 7.4 with hydrochloric acid. The temperature was maintained at $37 \pm 1{ }^{\circ} \mathrm{C}$ during the dissolution.

\subsubsection{Drug Distribution in GIT}

Male Sprague-Dawley rats (200-250 g) were fasted for 18-20 $\mathrm{h}$ before the commencement of the experiment. Rats were divided into 5 groups with 15 rats in each group. The first group (control) received oral administration of an aqueous solution of sitagliptin. The second to fifth groups received sitagliptin nanoparticles. Oral administration of nanoparticles was performed by diluting $20 \mathrm{mg}$ 
of nanoparticles in $1.0 \mathrm{~mL}$ saline, and injected using a rubber tube without anesthesia. Three rats were sacrificed each after 1, 3, 5, 8, and $12 \mathrm{~h}$ of administration. Stomach (Section 1) along with the entire small intestine were isolated and further subdivided into six sections (Sections 2-7; length of each section, $14 \mathrm{~cm}$ ). The obtained stomach and intestinal sections were then cut open to expose the inner mucosal surface. All nanoparticles located in each part of the section were then harvested using a spatula to scratch the nanoparticles off the mucosa. The mixture was then mashed using a homogenizer to extract sitagliptin, and the solution was then left for $24 \mathrm{~h}$ before centrifuged at $800 \times \mathrm{g}$ for $20 \mathrm{~min}$, and the supernatant was then determined using a spectrophotometer.

\subsubsection{Data Analysis}

Response surface methodology (RSM) was used for analyzing factors in models where the response of interest to factors is non-linear and inclined by several variables. The mathematical modeling was analyzed using the Deign Exper V.10 software (Statease Inc., Minneapolis, MN, USA software). Statistical analysis was done using the SPSS software. Student's $t$-test was used for single comparison and a $p$-value $<0.05$ was considered as significant.

\section{Results and Discussion}

A central composite design was created to assess the effect and interaction of potential factors that play a role on the mucoadhesive nanoparticles of sitagliptin (MNS) percentage yield (Table 1). Twenty formulations of nanoparticles were prepared (Table 2) to identify the optimal conditions for nanoparticle preparation. Experiments were conducted to evaluate the interaction in random sequence in a face-centered manner, according to our previous study [17]. The response surface graph indicates the percentage yield of the mucoadhesive sitagliptin nanoparticle was between $64.5-91.25 \%$ (Table 1), and was not affected by transformations. The following quadratic setup suggests that the response measured is depending on three factors, albumin alcohol concentration (albumin, $\mathrm{g}$ ), inlet temperature $\left({ }^{\circ} \mathrm{C}\right)$, and feed flow rate $(\mathrm{mL} / \mathrm{h})$. As a result, fixing the optimal formulation will maximize the efficacy of nanoparticle yield, and minimize the effect of other factors in arriving at this point.

The polynomial equation between each factor giving the mathematical relationship in coded factor (Table 3) is:

The numerical optimization study was optimized to identify the accuracy of the experimental model to predict each variable factor in correlation to the measured surface responses. Ten optimum solutions were calculated and analyzed (Table 4) in a double-blind manner, based on the design space in this study. The optimized sitagliptin nanoparticle formulation was then selected, based on which numerical optimization equation can predict the closest results to the actual results. Then, three $(3,6,10)$ were randomly selected and investigated for the percentage yield response (Table 4). Data suggest that the percentage yield response was consistent with the response predicted by the design expert software. 
Table 1. Design summary of sitagliptin nanoparticles.

\begin{tabular}{|c|c|c|c|c|c|c|c|c|c|}
\hline Study Type & \multicolumn{2}{|c|}{ Response Surface } & \multicolumn{3}{|c|}{20} & & & & \\
\hline Initial Design & \multicolumn{2}{|c|}{ Central Composite } & Blocks & \multicolumn{2}{|c|}{ No Blocks } & & & & \\
\hline Design Model & \multicolumn{2}{|c|}{ Quadratic } & & & & & & & \\
\hline Factor & Name & Units & Type & Low Actual & High Actual & Low Coded & High Coded & Mean & Std. Dev. \\
\hline A & Albumin Conc & $\%$ & Numeric & 1 & 2 & -1 & 1 & 1.5 & 0.353 \\
\hline $\mathrm{B}$ & Inlet Temperature & degree & Numeric & 100 & 120 & -1 & 1 & 110 & 7.071 \\
\hline $\mathrm{C}$ & Feed Flow rate & $\%$ & Numeric & 5 & 25 & -1 & 1 & 15 & 7.071 \\
\hline Response & Name & Units & Obs & Analysis & Minimum & Maximum & Mean & Std. Dev. & Ratio \\
\hline $\mathrm{Y} 1$ & Percentage yield & $\%$ & 20 & Polynomial & 64.5 & 91.25 & 75.658 & 7.25 & 1.41 \\
\hline
\end{tabular}


The effect of the three resultant factors, including albumin concentration (albumin, g), feed flow rate $(\mathrm{mL} / \mathrm{h})$, and inlet temperature $\left({ }^{\circ} \mathrm{C}\right)$ on the nanoparticle percentage yield response was then assessed (Table 5). Data concluded that the albumin concentration and inlet temperature have a significant effect on the percentage yield of the nanoparticles. ANOVA for the response surface model (Table 5) suggests that a lower inlet temperature and a higher albumin concentration resulted in a higher percentage yield (Figure 1), which is consistent with the earlier reports [18]. The relationship between the dependent (albumin concentration and inlet temperature) and independent (feed flow rate) variables on the percentage yield was further investigated using the response surface graph plots. However, data indicate that the nanoparticle percentage yield was higher when the feed flow rate was low (Figure 2), and the correlation coefficient was $\sim 0.59$. Although the spray rate was not the only factor that was varied, the collection of nanoparticles by hand could also be contributing to this varied response. Furthermore, a decrease in both feed flow rate and inlet temperature leads to a decrease in the sitagliptin nanoparticle percentage yield (Figure 3). Thus, to minimize the loss of sitagliptin nanoparticles during cyclone collection, the concentration of albumin polymer solution was increased to form a nanoparticle with a denser structure, resulting in a higher percentage yield.

Table 2. Three-variable central composite design of nanoparticles.

\begin{tabular}{cccccc}
\hline Run & Design Point & Albumin Conc. & Inlet Temp. & Flow rate & Percentage Yield \\
\hline 1 & Fact & 1 & 100 & 5 & 70.15 \\
5 & Fact & 1 & 120 & 5 & 64.5 \\
8 & Fact & 1 & 100 & 25 & 69.5 \\
19 & Fact & 1 & 120 & 25 & 79.5 \\
15 & Axial & 1 & 110 & 15 & 67.4 \\
18 & Axial & 1.5 & 100 & 15 & 83.1 \\
10 & Axial & 1.5 & 120 & 15 & 70.32 \\
14 & Axial & 1.5 & 110 & 5 & 67.1 \\
6 & Axial & 1.5 & 110 & 25 & 70.1 \\
20 & Center & 1.5 & 110 & 15 & 75 \\
3 & Center & 1.5 & 110 & 15 & 80 \\
7 & Center & 1.5 & 110 & 15 & 81.24 \\
12 & Center & 1.5 & 110 & 15 & 79.1 \\
2 & Center & 1.5 & 110 & 15 & 80.2 \\
11 & Center & 1.5 & 110 & 15 & 80.1 \\
4 & Fact & 2 & 100 & 5 & 85.7 \\
13 & Fact & 2 & 120 & 5 & 65.8 \\
16 & Fact & 2 & 100 & 25 & 91.25 \\
9 & Fact & 2 & 120 & 25 & 71.9 \\
17 & Axial & 2 & 110 & 15 & 81.2 \\
\hline
\end{tabular}

Table 3. Coefficients in terms of coded factor

\begin{tabular}{cc}
\hline Percentage Yield & $=$ \\
\hline 75.658 & $* \mathrm{~A}$ \\
4.48 & $* \mathrm{~B}$ \\
-4.768 & $* \mathrm{C}$ \\
2.9 & $* \mathrm{~A} * \mathrm{~B}$ \\
-5.45 & $* \mathrm{~A} * \mathrm{C}$ \\
-0.3375 & $* \mathrm{~B} * \mathrm{C}$ \\
2.025 & \\
\hline
\end{tabular}


Table 4. Optimization solutions for sitagliptin nanoparticles.

\begin{tabular}{lccccc}
\hline & Albumin Conc. $\mathbf{g}$ & Inlet Temp. ${ }^{\circ} \mathbf{C}$ & $\begin{array}{c}\text { Flow Rate } \\
\text { (Rounded to 0.5) } \mathbf{~ m L / h}\end{array}$ & Predicted & Actual \\
\hline Suggestion-1 & 2 & 106.65 & 25 & 85.45 & 84.51 \\
Suggestion-2 & 2 & 106.56 & 25 & 85.52 & 85.12 \\
Suggestion-3 & 2 & 106.75 & 25 & 85.37 & 84.91 \\
Suggestion-4 & 2 & 106.56 & 24.71 & 85.46 & 84.60 \\
Suggestion-5 & 1.99 & 106.69 & 25 & 85.35 & 86.1 \\
Suggestion-6 & 1.99 & 106.59 & 25 & 85.42 & 87.1 \\
Suggestion-7 & 2 & 106.73 & 24.44 & 85.27 & 85.12 \\
Suggestion-8 & 1.99 & 106.43 & 25 & 85.49 & 85.46 \\
Suggestion-9 & 2 & 107.71 & 25 & 84.57 & 83.98 \\
Suggestion-10 & 2 & 106.66 & 24.08 & 85.27 & 84.69 \\
\hline
\end{tabular}

Table 5. ANOVA for response surface model.

\begin{tabular}{cccccc}
\hline Source & Sum of Squares & df & Mean Square & $\boldsymbol{F}$ Value & Prob $>\boldsymbol{F}$ \\
\hline Model & 783.4785 & 6 & 130.5797 & 6.313662 & 0.0027 \\
A-Albumin Conc & 200.704 & 1 & 200.704 & 9.70424 & 0.0082 \\
B-Inlet Temperature & 227.3382 & 1 & 227.3382 & 10.99203 & 0.0056 \\
C-Feed Flow Rate & 84.1 & 1 & 84.1 & 4.066319 & 0.0649 \\
AB & 237.62 & 1 & 237.62 & 11.48917 & 0.0048 \\
AC & 0.91125 & 1 & 0.91125 & 0.04406 & 0.8370 \\
BC & 32.805 & 1 & 32.805 & 1.586155 & 0.2300 \\
\hline
\end{tabular}

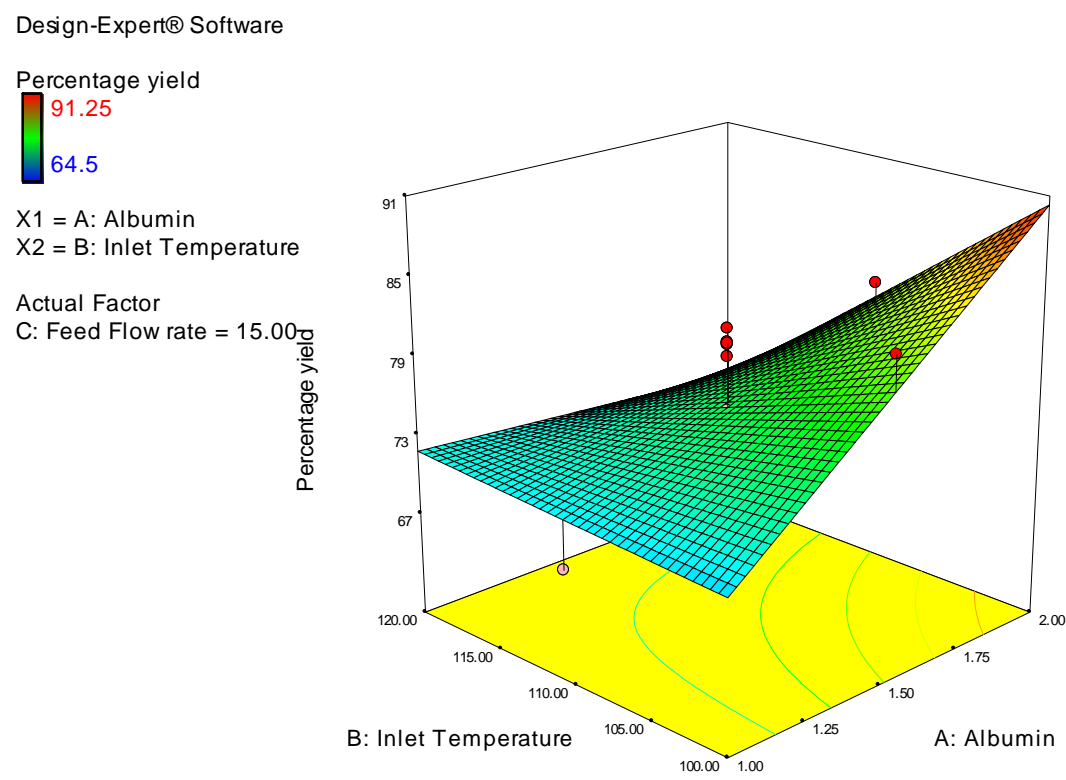

Figure 1. Response surface graph showing the effect on inlet temperature vs albumin on percentage yield. 


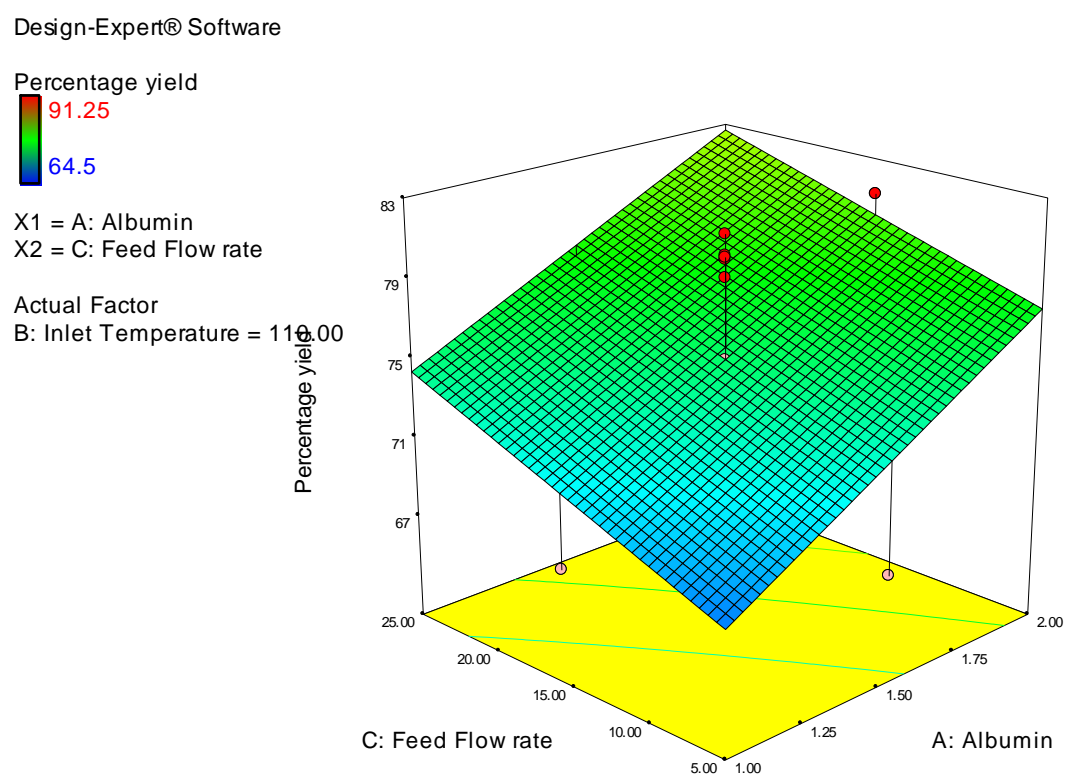

Figure 2. Response surface graph showing the effect on feed flow rate vs albumin on percentage yield.

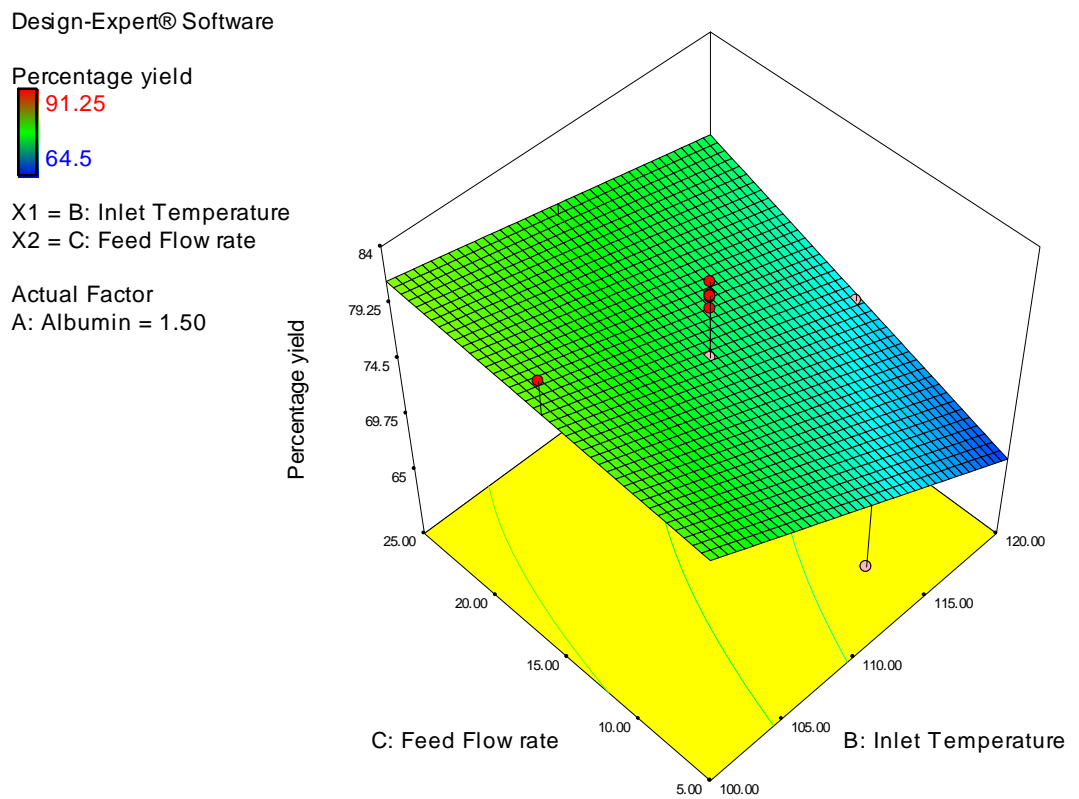

Figure 3. Response surface graph showing the effect on feed flow rate vs inlet temperature on percentage yield.

Mucoadhesive nanoparticles of sitagliptin (MNS) had different surface texture and drug loading according to different experimental formulations. A drug loading percentage of $73 \pm 2 \%$ and a nanoparticle percentage yield of $64-92 \%$ for $500 \mathrm{mg}$ powder was assessed. Eight out of the 20 experiments indicate the recovery rate of the nanoparticle was higher than $80 \%$. It is predicted that the loss of products during drug loading happened due to the residual nanoparticles being attached to the spraying chamber, or by the loss of nanoparticles from the manual electrode collection.

Nanoparticles prepared from the optimized experimental conditions were nearly spherical, and were moving in a continuous and steady stream. Examination using SEM microphotographs did not show any shriveled/surface folding shape (Figure 4). This may be due to the consistency during the drying period of the nanoparticle in the spraying column. Furthermore, the change in the nanoparticle shape during spray drying is possibly related to moisture variation in the material content 
and the spray drying temperature. The small particles may dry faster than the larger ones, and the important step of the crust formation on the surface of the nanoparticles was reached immediately after atomization. This problem was consistent and was overcome from our previous experiments.

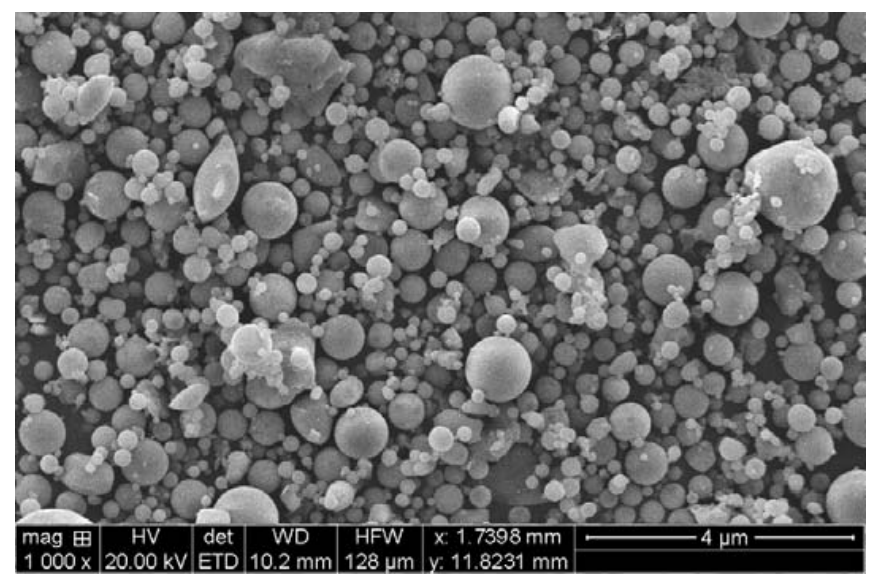

Figure 4. SEM image of nanoparticles used in this study.

Our data indicate that the average size of the sitagliptin nanoparticles (Figure 5) is approximately $659 \mathrm{~nm}$, although sizes were spread, from 350 to $950 \mathrm{~nm}$. The reduced and narrowed nanoparticle size can facilitate a near immediate sitagliptin release at the absorption site, and is highly deliberated, thus, with more retention of a larger amount of sitagliptin. This may, in turn, contribute to the hypoglycemic effects of sitagliptin.

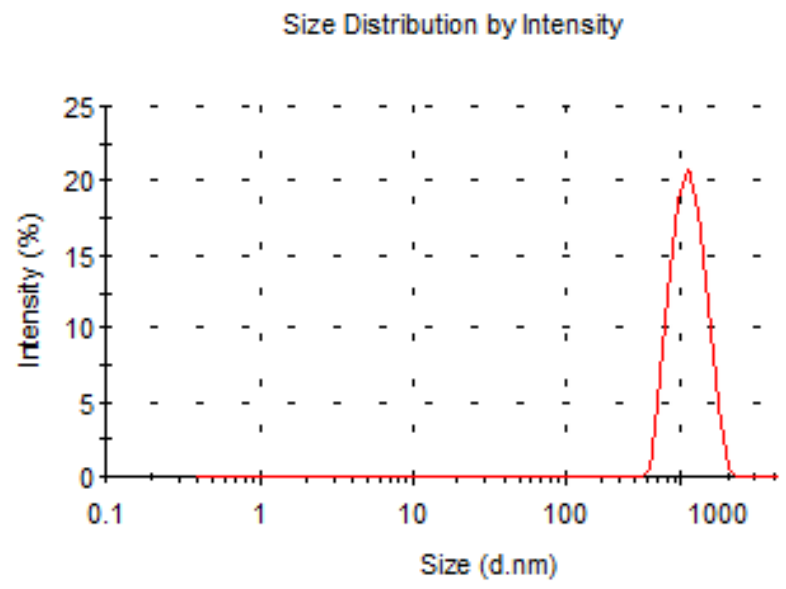

Figure 5. Particle size distribution of sitagliptin nanoparticles.

The percentage of sitagliptin nanoparticles formulation swelling with time was also observed (Figure 6). All nanoparticles formulations swelled rapidly within $0.1 \mathrm{~h}$ when immersed in phosphate buffered saline ( $\mathrm{pH}$ 7.4). It was stated that the mucoadhesive properties of the nanoparticle and the cohesiveness of the particle polymers could be affected by the swelling index [19]. Mucoadhesive nanoparticles are expected to absorb liquid from the stomach mucosal tissue. Furthermore, swelling of the nanoparticles and the capillary effects of the mucosal tissue lead to a stronger mucoadhesion. The percent swelling of the MNS formulation is $168 \pm 15 \%$ (Figure 6). 


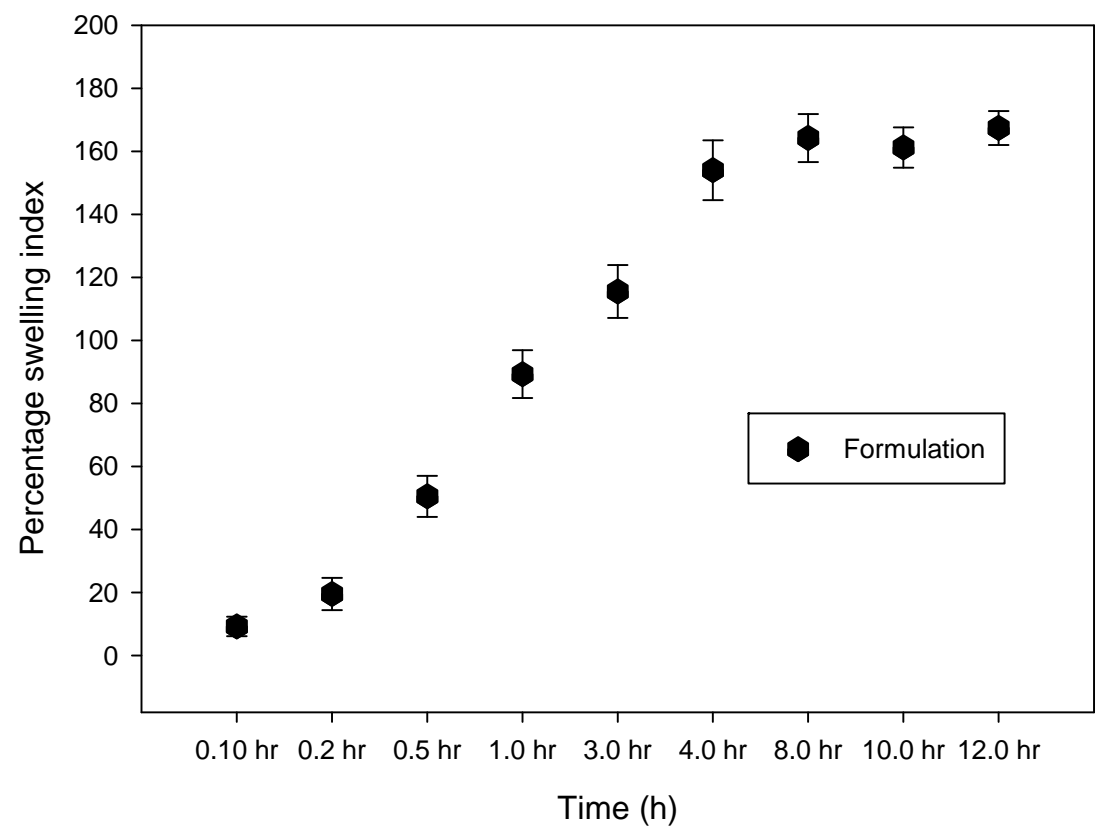

Figure 6. Swelling measurement of nanoparticle formulation.

Bioadhesion studies were then performed to investigate the mucoadhesive properties of the nanoparticles. Our data indicate that the mucoadhesion of sitagliptin nanoparticles $(6.1 \pm 0.5 \mathrm{~h})$ is excellent (ex vivo). The adhesion between nanoparticles and mucin (the glycoprotein predominant in the mucosal tissue) is primarily due to electrostatic forces, the functional carboxyl groups on the sitagliptin nanoparticles, and the hydrogen bonds in the mucus molecules, creating adhesive forces between the nanoparticle polymers. This characterized mucoadhesive property of the sitagliptin nanoparticles then lead to the study of sitagliptin release at the gastrointestinal tract (GIT) from the nanoparticles. However, in the control group (sitagliptin in drug solution), sitagliptin was splashed out straight away from the rat intestinal mucosa, indicating a lack of mucoadhesive property.

Sitagliptin nanoparticles were then assessed for in vitro release (Figure 7). Approximately $41.5 \%$ of the total sitagliptin in mucoadhesive nanoparticles was released within the first hour of delivery, suggesting that sitagliptin was adsorbed or fused from the surface of the nanoparticles significantly at the beginning of the delivery. This could be explained by a prime "burst effect" in the clinical setting that facilitates the optimized nanoparticle formulation to be released, and has a fast effect in patients. Nevertheless, the sitagliptin discharged from the albumin nanoparticles was retained up to $12 \mathrm{~h}(99.1 \%)$, indicating the mucoadhesive nanoparticles have the potential for controlled drug release. On the contrary, sitagliptin was rapidly released-up to $91.5 \%$ in the first half hour from the control group. These results indicate a controlled and efficient release of the sitagliptin nanoparticles. The data obtained from this in vitro sitagliptin release from nanoparticles studies are then compared using different kinetic models, including first-order, Higuchi, Weibull, Baker and Lonsdale, Hixson and Crowell, and Korsmeyer-Peppas [17]. A macro written graphing tool (SigmaPlot version 9.01) was used to study the mechanism of the nanoparticle drug release and the release rate. The correlation coefficient value $\left(R^{2}\right)$ was also considered when selecting a significant model or curve fit that would best to investigate the extent of the sitagliptin release. Data suggest that Korsmeyer-Peppas model is an ideal fit, with $R^{2}=0.994$ (Figure 7). According to the Korsmeyer-Peppas fit, the release of the drug, sitagliptin, from the nanoparticles, is through the polymeric matrix, and follows Fick's law of diffusion. The release pattern of mucoadhesive sitagliptin nanoparticles according to the classical Fick's diffusion, together with the initial swelling of the nanoparticles and the early drug adsorption on the surface of the nanoparticle, possibly contribute to the "burst" release [20]. After this "burst" effect, the sitagliptin release from the nanoparticle pattern is better controlled, and maintained for longer period of time. 


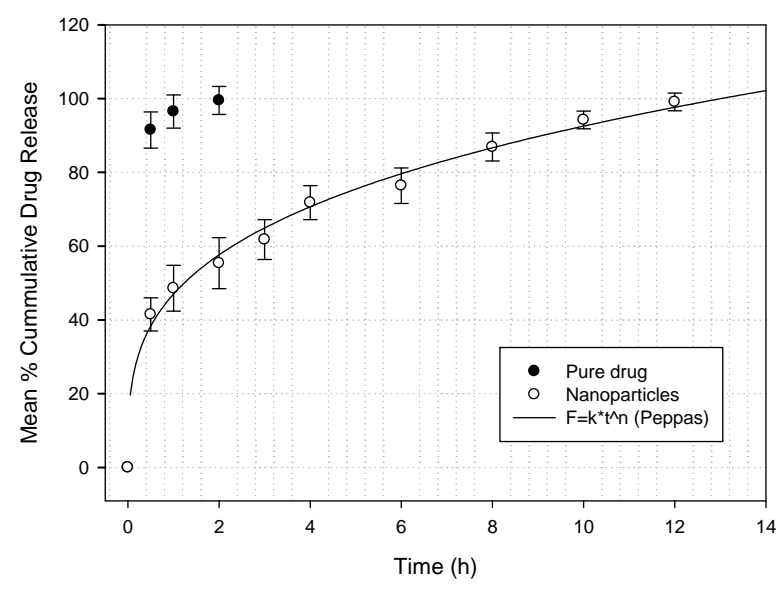

Figure 7. In vitro release studies of sitagliptin nanoparticles.

To assess the percentage drug concentration, sitagliptin suspension and MNS nanoparticles were orally ingested into Sprague-Dawley rats, as previously described. The mucoadhesiveness of the nanoparticles were examined at $30 \mathrm{~min}, 1 \mathrm{~h}, 4 \mathrm{~h}, 6 \mathrm{~h}, 8 \mathrm{~h}$, and $12 \mathrm{~h}$ in vivo. The remaining percentage of MNS nanoparticles in the gastrointestinal tract (GIT) was significantly higher than that of the sitagliptin suspension $(p<0.05)$. The results indicated that polymer was incorporated in the nanoparticles due to their chemical components and the readily swollen large mass polymers in PBS, providing a large adhesive surface on the nanoparticle for the maximum interaction with the mucin, which prolonged the residence time of the nanoparticles in the GIT. Also, perhaps hydrogen bonding is a key part of the adhesion [21,22]. The albumin polymers adhere better in an acidic environment and are in a highly protonated condition, possibly due to the presence of a large number of carboxylic groups to form hydrogen bonds. MNS showed percentage of drug release at $30 \mathrm{~min}$ is $12 \%, 1 \mathrm{~h}-32 \%, 4 \mathrm{~h}-64 \%$, $6 \mathrm{~h}-78 \%, 8 \mathrm{~h}-87 \%$, and $12 \mathrm{~h}-97 \%$ (Figure 8 ). In comparison with sitagliptin nanoparticles, the amount of drug that was released at $30 \mathrm{~min}$ was $54 \%$, and at $1 \mathrm{~h}$ the total drug released was $98 \%$. At the same time, MNS nanoparticles showed 32\% drug release, indicating a significant amount of sitagliptin was adsorbed on or incorporated at the surface of the nanoparticles. This may be due to the drug's "burst effect" and allows the nanoparticle preparation to have a fast and effective effect on the patients in clinical practice.

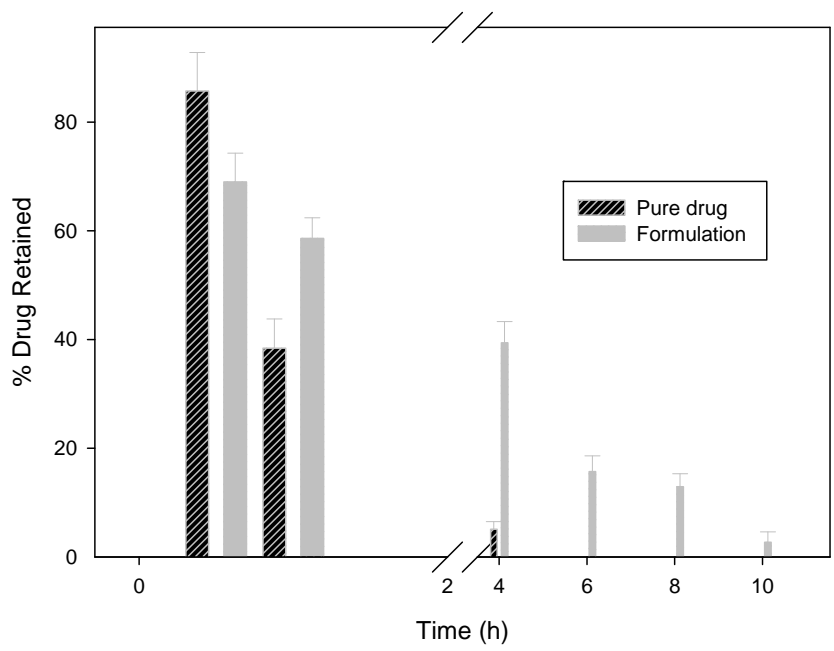

Figure 8. Percentage drug retained in the stomach after oral administration of formulation and pure drug. 


\section{Conclusions}

This study concludes that an optimized mucoadhesive nanoparticle formulation was developed to extend the retention and release time of the antidiabetic drug sitagliptin. The described optimized mucoadhesive nanoparticles are a first and could be an ideal drug delivery system for the effective delivery of sitagliptin for treating type 2 diabetic patients.

Author Contributions: A.H.A., S.H. and B.E.A.-D. conceived and designed the experiments; A.H.A., S.H., N.H.P. and B.E.A.-D. performed the experiments; A.H.A., S.H., N.H.P. and B.E.A.-D. analyzed the data; A.H.A. and S.H., contributed reagents/materials/analysis tools; S.H. wrote the paper.

Acknowledgments: I gratefully thank, for the research funding from Deanship of Scientific Research, King Faisal University, Al-Ahsa, Saudi Arabia. Grant Number: 160148.

Conflicts of Interest: The authors declare no conflict of interest.

\section{References}

1. Sivaprasad, S.; Gupta, B.; Crosby-Nwaobi, R.; Evans, J. Prevalence of Diabetic Retinopathy in Various Ethnic Groups: A Worldwide Perspective. Surv. Ophthalmol. 2012, 57, 347-370. [CrossRef] [PubMed]

2. Dong, H.-Q.; Li, M.; Zhu, F.; Liu, F.-L.; Huang, J.-B. Inhibitory potential of trilobatin from Lithocarpus polystachyus Rehd against $\alpha$-glucosidase and $\alpha$-amylase linked to type 2 diabetes. Food Chem. 2011, 130, 261-266. [CrossRef]

3. Kikuchi, M.; Haneda, M.; Koya, D.; Tobe, K.; Onishi, Y.; Couturier, A.; Mimori, N.; Inaba, Y.; Goodman, M. Efficacy and tolerability of vildagliptin as an add-on to glimepiride in Japanese patients with Type 2 diabetes mellitus. Diabetes Res. Clin. Pract. 2010, 89, 216-223. [CrossRef] [PubMed]

4. Boutayeb, A.; Boutayeb, S. The burden of non communicable diseases in developing countries. Int. J. Equity Health 2005, 4, 2. [CrossRef] [PubMed]

5. Farag, Y.M.K.; Gaballa, M.R. Diabesity: An overview of a rising epidemic. Nephrol. Dial. Transpl. 2011, 26, 28-35. [CrossRef] [PubMed]

6. Kheir, N.; Zaidan, M.; Younes, H.; Hajj, M.E.; Wilbur, K.; Jewesson, P.J. Pharmacy Education and Practice in 13 Middle Eastern Countries. Am. J. Pharm. Educ. 2009, 72, 133. [CrossRef]

7. Elhadd, T.A.; Al-Amoudi, A.A.; Alzahrani, A.S. Epidemiology, clinical and complications profile of diabetes in Saudi Arabia: A review. Am. J. Pharm. Educ. 2007, 27, 241-250.

8. Al-Khader, A.A. Impact of diabetes in renal diseases in Saudi Arabia. Nephrol Dial Transplant. 2001, 16, 2132-2135.

9. Kikuchi, M.; Abe, N.; Kato, M.; Terao, S.; Mimori, N.; Tachibana, H. Vildagliptin dose-dependently improves glycemic control in Japanese patients with type 2 diabetes mellitus. Diabetes Res. Clin. Pract. 2009, 83, 233-240. [CrossRef] [PubMed]

10. Ahrén, B.; Schweizer, A.; Dejager, S.; Villhauer, E.B.; Dunning, B.E.; Foley, J.E. Mechanisms of action of the dipeptidyl peptidase-4 inhibitor vildagliptin in humans. Diabetes Obes. Metab. 2011, 13, 775-783. [CrossRef] [PubMed]

11. Noh, Y.-H.; Lim, H.-S.; Jin, S.-J.; Kim, M.J; Kim, Y.H.; Sung, H.R.; Choi, H.Y.; Bae, K.-S. Effects of Ketoconazole and Rifampicin on the Pharmacokinetics of Gemigliptin, a Dipeptidyl Peptidase-IV Inhibitor: A Crossover Drug-Drug Interaction Study in Healthy Male Korean Volunteers. Clin. Ther. 2012, 34, 1182-1194. [CrossRef] [PubMed]

12. Holst, J.J.; Deacon, C.F. Inhibition of the activity of dipeptidyl-peptidase IV as a treatment for type 2 diabetes. Diabetes 1998, 47, 1663-1670. [CrossRef] [PubMed]

13. Seewoodhary, J.; Bain, S.C. Novel treatments for type 2 diabetes. Br. J. Gen. Pract. 2011, 61, 5-6. [CrossRef] [PubMed]

14. Gupta, S.; Samanta, M.; Raichur, A. Dual-Drug Delivery System Based on In Situ Gel-Forming Nanosuspension of Forskolin to Enhance Antiglaucoma Efficacy. AAPS PharmSciTech 2010, 11, 322-335. [CrossRef] [PubMed]

15. Singh, B.; Chakkal, S.K.; Ahuja, N. Formulation and Optimization of Controlled Release Mucoadhesive Tablets of Atenolol Using Response Surface Methodology. AAPS Pharm Sci Tech 2006, 7, E19-E28. [CrossRef] [PubMed] 
16. Ponchel, G.; Irache, J.-M. Specific and non-specific bioadhesive particulate systems for oral delivery to the gastrointestinal tract. Adv. Drug Deliv. Rev. 1998, 34, 191-219. [CrossRef]

17. Harsha, N.S.; Rani, R.H.S. Drug targeting to lungs by way of microspheres. Arch. Pharm. Res. 2006, 29, 598-604. [CrossRef] [PubMed]

18. Harsha, S.; Attimard, M.; Khan, T.A.; Nair, A.B.; Aldhubiab, B.E.; Sangi, S.; Shariff, A. Design and formulation of mucoadhesive microspheres of sitagliptin. J. Microencapsul. 2013, 30, 257-264. [CrossRef] [PubMed]

19. Khonsari, F.; Zakeri-Milani, P.; Jelvehgari, M. Formulation and Evaluation of In-vitro Characterization of Gastic-Mucoadhesive Microparticles/Discs Containing Metformin Hydrochloride. Iran. J. Pharm. Res. 2014, 13, 67-80. [PubMed]

20. Singh, R.; Lillard, J.W. Nanoparticle-based targeted drug delivery. Exp. Mol. Pathol. 2009, 86, $215-223$. [CrossRef] [PubMed]

21. Dhaliwal, S.; Jain, S.; Hardevinder, P.S.; Tiwary, A.K. Mucoadhesive Microspheres for Gastroretentive Delivery of Acyclovir In Vitro and In Vivo Evaluation. AAPS J. 2004, 10, 322-330. [CrossRef] [PubMed]

22. Tao, Y.; Lu, Y.; Sun, Y.; Gu, B.; Lu, W.; Pan, J. Development of mucoadhesive microspheres of acyclovir with enhanced bioavailability. Int. J. Pharm. 2009, 378, 30-36. [CrossRef] [PubMed]

(C) 2018 by the authors. Licensee MDPI, Basel, Switzerland. This article is an open access article distributed under the terms and conditions of the Creative Commons Attribution (CC BY) license (http:/ / creativecommons.org/licenses/by/4.0/). 